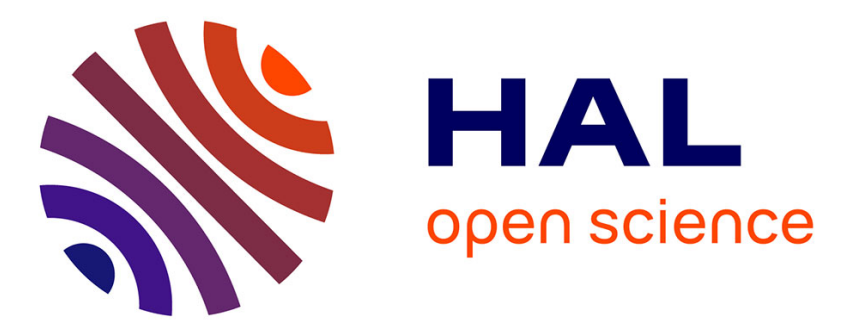

\title{
On Set-Membership Observer Design for a Class of Periodical Time-Varying Systems
}

\author{
Denis Efimov, Tarek Raissi, Stanislav Chebotarev, Ali Zolghadri
}

\section{To cite this version:}

Denis Efimov, Tarek Raissi, Stanislav Chebotarev, Ali Zolghadri. On Set-Membership Observer Design for a Class of Periodical Time-Varying Systems. 51st IEEE Conference on Decision and Control 2012, Dec 2012, Hawaii, United States. hal-00745505

\section{HAL Id: hal-00745505 \\ https://hal.inria.fr/hal-00745505}

Submitted on 26 Oct 2012

HAL is a multi-disciplinary open access archive for the deposit and dissemination of scientific research documents, whether they are published or not. The documents may come from teaching and research institutions in France or abroad, or from public or private research centers.
L'archive ouverte pluridisciplinaire HAL, est destinée au dépôt et à la diffusion de documents scientifiques de niveau recherche, publiés ou non, émanant des établissements d'enseignement et de recherche français ou étrangers, des laboratoires publics ou privés. 


\title{
On Set-Membership Observer Design for a Class of Periodical Time-Varying Systems
}

\author{
Denis Efimov $^{\dagger}$, Tarek Raïssi ${ }^{2}$, Stanislav Chebotarev ${ }^{\ddagger}$, Ali Zolghadri ${ }^{\natural}$
}

\begin{abstract}
This work is devoted to interval observer design for Linear Time Varying (LTV) systems and a class of nonlinear time-varying systems in the output canonical form. An interval observer design is feasible if it is possible to calculate the observer gains making the estimation error dynamics cooperative and stable. It has been shown that under some mild conditions the cooperativity of an LTV system can be ensured by a static linear transformation of coordinates. The case of a timevarying transformation for periodic systems is considered in this work. The efficiency of the proposed approach is demonstrated through computer simulations.
\end{abstract}

\section{INTRODUCTION}

The problem of unmeasurable state vector estimation is very challenging and its solution is demanded in many applications [6], [9], [20]. In some situations due to the presence of uncertainty (parametric or/and signal) the design of a conventional estimator, converging in the noise-free case to the ideal value of the state, is not possible. However, an interval estimation remains feasible. By interval or setmembership estimation we understand an observer that, using input-output information, evaluates the set of admissible values (interval) for the state at each instant of time.

There are several approaches to design interval observers [3], [11], [13], [18]. This paper continues the framework of interval observer design based on the monotone system theory [3], [18]. Such an approach has been recently extended in [22] to nonlinear systems using a LPV representation with known minorant and majorant matrices, in [21] for observable nonlinear systems and in [8] for a combined application of interval and sliding-mode observers. One of the most complex assumptions for the interval observer design, dealing with cooperativity of the interval estimation error dynamics, was relaxed in [16], [21]. It was shown that under

\footnotetext{
${ }^{\dagger}$ Non-A project at INRIA - LNE, Parc Scientifique de la Haute Borne, 40 avenue Halley, Bât.A Park Plaza, 59650 Villeneuve d'Ascq, France, denis.efimov@inria.fr.

'Conservatoire National des Arts et Métiers (CNAM), Département EASY, Cedric - laetitia 292, Rue St-Martin, case 2D2P10, 75141 Paris Cedex 03, tarek.raissi@cnam.fr.

${ }^{\ddagger}$ Department of Control Systems and Informatics, Saint Petersburg State University of Information Technologies Mechanics and Optics, Kronverkskiy av. 49, Saint Petersburg, 197101, Russia.

GUniversity of Bordeaux, IMS-lab, Automatic control group, 351 cours de la libération, 33405 Talence, France, Ali.Zolghadri@ims-bordeaux.fr.

This work is partially supported by financial support of FGP "Research and scientific-pedagogical personnel of innovative Russia" for 2009 - 2013 years (the agreement 14.B37.21.0875).
}

some mild conditions applying similarity transformation, a Hurwitz matrix could be transformed to Hurwitz and Metzler one (cooperative). The transformation matrix is a solution of the Sylvester equation, a constructive procedure for this solution calculation was also given in [21].

In order to apply the approach of interval observer design to the systems with non-constant matrices dependent on measurable input-output signals and time, an extension of the result from [21] has been presented in [7], which allows one to calculate a constant similarity transformation matrix representing a given interval of matrices to an interval of Metzler matrices (this result is introduced in Section 3 for completeness and comparison). This method can be used to design interval observers for Linear-Parameter-Varying (LPV) systems [17], [23], [25] with measurable vector of scheduling parameters.

An important class of time-varying systems is constituted by periodical ones [4], [5], [26], [27]. For periodic systems a time-varying transformation of coordinates is proposed in this work using the Floquet theory, which represents the system in a time-invariant cooperative form. Simulations of an example of such systems are considered in this work to demonstrate the proposed solution efficiency.

The paper is organized as follows. Some basic facts from the theory of interval estimation are given in Section 2. The main result is described in Section 3. The example of computer simulation is presented in Section 4.

\section{Preliminaries}

Euclidean norm for a vector $x \in \mathbb{R}^{n}$ will be denoted as $|x|$, and for a measurable and locally essentially bounded input $u: \mathbb{R}_{+} \rightarrow \mathbb{R}\left(\mathbb{R}_{+}=\{\tau \in \mathbb{R}: \tau \geq 0\}\right)$ the symbol $\|u\|_{\left[t_{0}, t_{1}\right]}$ denotes its $L_{\infty}$ norm:

$$
\|u\|_{\left[t_{0}, t_{1}\right]}=e s s \sup \left\{|u(t)|, t \in\left[t_{0}, t_{1}\right]\right\},
$$

if $t_{1}=+\infty$ then we will simply write $\|u\|$. We will denote as $\mathcal{L}_{\infty}$ the set of all inputs $u$ with the property $\|u\|<\infty$. Denote the sequence of integers $1, \ldots, k$ as $\overline{1, k}$. The symbols $I_{n}$ and $E_{n}$ denote the identity matrix and the matrix with all elements equal 1 respectively (with dimension $n \times n$ ). For a matrix $A \in \mathbb{R}^{n \times n}$ the vector of its eigenvalues is denoted as $\lambda(A), \|\left. A\right|_{\max }=\max _{i=\overline{1, n}, j=\overline{1, n}}\left|A_{i, j}\right|$ (the elementwise maximum norm, it is not sub-multiplicative) and $\|A\|_{2}=\sqrt{\max _{i=1, n} \lambda_{i}\left(A^{\mathrm{T}} A\right)}$ (the induced $L_{2}$ matrix 
norm), the relation $\|A\|_{\max } \leq\|A\|_{2} \leq n\|A\|_{\max }$ is satisfied between these norms.

\section{A. Cooperative systems and interval arithmetic}

For two vectors $x_{1}, x_{2} \in \mathbb{R}^{n}$ or matrices $A_{1}, A_{2} \in \mathbb{R}^{n \times n}$, the relations $x_{1} \leq x_{2}$ and $A_{1} \leq A_{2}$ are understood elementwise. The relation $P \succ 0$ means that the matrix $P \in \mathbb{R}^{n \times n}$ is positive definite. Given a matrix $A \in \mathbb{R}^{m \times n}$ define $A^{+}=\max \{0, A\}, A^{-}=A^{+}-A$ (similarly for vectors).

Lemma 1. Let $x \in \mathbb{R}^{n}$ be a vector variable, $\underline{x} \leq x \leq \bar{x}$ for some $\underline{x}, \bar{x} \in \mathbb{R}^{n}$, and $A \in \mathbb{R}^{m \times n}$ be a constant matrix, then

$$
A^{+} \underline{x}-A^{-} \bar{x} \leq A x \leq A^{+} \bar{x}-A^{-} \underline{x} .
$$

Proof: For $\underline{x} \leq x \leq \bar{x}$ we have $A x=\left(A^{+}-A^{-}\right) x$ that gives the required estimates.

A matrix $A \in \mathbb{R}^{n \times n}$ is called Hurwitz if all its eigenvalues have negative real parts, it is called Metzler if all its elements outside the main diagonal are nonnegative. Any solution of the linear system

$$
\dot{x}=A x+\omega(t), \omega: \mathbb{R}_{+} \rightarrow \mathbb{R}_{+}^{n},
$$

with $x \in \mathbb{R}^{n}$ and a Metzler matrix $A$, is elementwise nonnegative for all $t \geq 0$ provided that $x(0) \geq 0$ [24]. Such dynamical systems are called cooperative (monotone) [24].

Lemma 2. [21] Given the matrices $A \in \mathbb{R}^{n \times n}, R \in \mathbb{R}^{n \times n}$ and $C \in \mathbb{R}^{p \times n}$. If there is a matrix $L \in \mathbb{R}^{n \times p}$ such that the matrices $A-L C$ and $R$ have the same eigenvalues, then there is a $S \in \mathbb{R}^{n \times n}$ such that $R=S(A-L C) S^{-1}$ provided that the pairs $\left(A-L C, \chi_{1}\right)$ and $\left(R, \chi_{2}\right)$ are observable for some $\chi_{1} \in \mathbb{R}^{1 \times n}, \chi_{2} \in \mathbb{R}^{1 \times n}$.

This result was used in [21] to design interval observers for linear time invariant systems with a Metzler matrix $R$ (the main difficulty is to prove the existence of a real matrix $S$, and to provide a constructive approach of its calculation).

\section{B. Floquet theory}

Consider a linear periodical time-varying system

$$
\dot{x}=A(t) x,
$$

where $x \in \mathbb{R}^{n}$ is the state and $A: \mathbb{R}_{+} \rightarrow \mathbb{R}^{n \times n}$ is a periodic piecewise continuous function with period $\tau>0$, i.e. $A(t)=A(t+\tau)$ for all $t \in \mathbb{R}_{+}$. Denote by $\Phi(t)$ the fundamental matrix of the system (2) (it is the solution of the matrix differential equation $\dot{\Phi}(t)=A(t) \Phi(t)$ for $t \geq 0$ and $\left.\Phi(0)=I_{n}\right)$, then $\Phi(t+\tau)=\Phi(t) H$, where $H$ is the monodromy matrix of the system. The Floquet representation provides that $\Phi(t)=W(t) e^{t B}$ for a $2 \tau$-periodical matrix function $W: \mathbb{R}_{+} \rightarrow \mathbb{R}^{n \times n}$ and some $B \in \mathbb{R}^{n \times n}$ [12], then $B=0.5 \tau^{-1} \ln H^{2}$ and $W(t)=\Phi(t) e^{-t B}$ for $0 \leq t \leq 2 \tau$. Introducing the transformation of coordinates $\xi=W(t)^{-1} x$ we obtain a time-invariant representation of the system (2):

$$
\dot{\xi}=B \xi \text {. }
$$

Since $W$ is continuous and periodical (it is bounded), then the system (2) stability follows from Hurwitz property of the matrix $B$.

\section{MAIN RESULT}

In this work we consider the following model of a nonlinear time-varying system:

$$
\begin{gathered}
\dot{x}=A(t, y, u) x+f(t, x, u, \varrho), \\
y=C(t, u) x,
\end{gathered}
$$

where $x \in \mathbb{R}^{n}, u \in \mathbb{R}^{m}, y \in \mathbb{R}^{p}$ are the state, the input and the output of the system (3), $\varrho \in \Theta \subset \mathbb{R}^{q}$ is the vector of unknown signals or parameters, the compact set $\Theta$ is given, the matrix functions $A: \mathbb{R}^{p+m+1} \rightarrow \mathbb{R}^{n \times n}, C: \mathbb{R}^{m+1} \rightarrow \mathbb{R}^{p \times n}$ and the function $f: \mathbb{R}^{n+m+q+1} \rightarrow \mathbb{R}^{n \times m}$ are given. The instant values of $u(t) \in \mathcal{L}_{\infty}, y(t) \in \mathcal{L}_{\infty}$ are known. In this work we consider the case without measurement noise, the proposed result can be extended to the case with a noise in the measurement channel, this extension is omitted for brevity of presentation. Denoting $\theta(t)=\left[\begin{array}{lll}t & y & u\end{array}\right]^{\mathrm{T}}$ we can rewrite the system (3) in the quasi-LPV form with a measurable scheduling parameter vector $\theta$.

Many works on the interval observer design [3], [18], [22], [21] deal with the case of a constant matrix $A$ (or under some transformations the estimation error can be represented in the form with a constant matrix $A$, next an observer gain $L$ can be found such that $A-L C$ is Hurwitz and Metzler). In the work [7] such a restriction has been avoided. First, to introduce that result we need the following assumptions.

Assumption 1. $\|x\| \leq X,\|u\| \leq U$ and $\|y\| \leq Y$, the constants $X>0, U>0$ and $Y>0$ are given.

Boundedness of the state $x$ and the input $u$ is a standard assumption in the estimation theory.

Assumption 2. Let $\underline{x} \leq x \leq \bar{x}$ for some $\underline{x} \in \mathbb{R}^{n}$ and $\bar{x} \in \mathbb{R}^{n}$, then $f(t, \underline{x}, \bar{x}, u) \leq f(t, x, u, \varrho) \leq \bar{f}(t, \underline{x}, \bar{x}, u)$ for some given $f: \mathbb{R}^{2 n+m+1} \rightarrow \mathbb{R}^{n}, \bar{f}: \mathbb{R}^{2 n+m+1} \rightarrow \mathbb{R}^{n}$ and all $t \geq 0,\|\bar{u}\| \leq U, \varrho \in \Theta$.

Assumption 3. There exist matrix functions $L: \mathbb{R}^{p+m+1} \rightarrow$ $\mathbb{R}^{n \times p}, P: \mathbb{R}_{+} \rightarrow \mathbb{R}^{n \times n}, P(\cdot)=P(\cdot)^{T} \succ 0$ such that for all $t \geq 0$ and $\|u\| \leq U,\|y\| \leq Y$ :

$$
\begin{gathered}
p_{1} I_{n} \preceq P(t) \preceq p_{2} I_{n}, p_{1}, p_{2}>0 ; \\
\dot{P}(t)+D(t, y, u)^{T} P(t)+P(t) D(t, y, u)+P(t)^{2}+Q=0, \\
D(t, y, u)=A(t, y, u)-L(t, y, u) C(t, u), Q=Q^{T} \succ 0 .
\end{gathered}
$$

Assumption 2 states that if the bounds $\underline{x}, \bar{x}$ on the state value $x$ are given, then the values of the nonlinear function $f$ are enclosed in the interval $[f, \bar{f}]$ for all $\varrho \in \Theta$ (for a continuous $f$, the computation of $f, \bar{f}$ for given $\underline{x}, \bar{x}$ and a convex $\Theta$ can be performed using the interval arithmetics [19], [14]). In assumption 3 the observer gain $L(t, y, u)$ is introduced, that ensures stability of the time-varying matrix 
$D(t, y, u)$ with the Lyapunov function matrix $P(t)$, this assumption determines the stability conditions of the estimation dynamics (if $D(t, y, u)=D(t)$, then this assumption is a conventional requirement for linear time-varying systems). Due to assumption 1 the matrix $A$ is varying in a compact domain, then Linear Parameter-Varying or polytopic system results [1], [2], [10], [15] can be used to compute a gain $L$ satisfying assumption 3 . If $D(t, y, u)=D(t)$, then this assumption is a conventional requirement for linear timevarying systems, if in addition $D(t)$ is periodical, then this inequality can be solved as a differential equation [26].

Under these assumptions, if we additionally assume that the matrix $D$ is Metzler, then the following interval observer can be designed [3], [18], [22]:

$$
\begin{aligned}
& \dot{x}=A(t, y, u) \underline{x}+\underline{f}(t, \underline{x}, \bar{x}, u)+L(t, y, u)[y-C(t, u) \underline{x}], \\
& \dot{\bar{x}}=A(t, y, u) \bar{x}+\bar{f}(t, \underline{x}, \bar{x}, u)+L(t, y, u)[y-C(t, u) \bar{x}] .
\end{aligned}
$$

Theorem 1. [7] Let assumptions 1, 2 and 3 hold, and the matrix $D(t, y, u)$ be Metzler for all $t \geq 0$ and $\|u\| \leq U$, $\|y\| \leq Y$. Let one of the following conditions be satisfied:

1) $|f(t, \underline{x}, \bar{x}, u)|<+\infty,|\bar{f}(t, \underline{x}, \bar{x}, u)|<+\infty$ for any $t \geq 0,\|u\| \leq U$ and all $\underline{x} \in \mathbb{R}^{n}, \bar{x} \in \mathbb{R}^{n}$;

2) for any $t \geq 0,\|x\| \leq X,\|u\| \leq U, \varrho \in \Theta$ and all $\underline{x} \in \mathbb{R}^{n}, \bar{x} \in \mathbb{R}^{n}$

$$
\begin{gathered}
|f(t, x, u, \varrho)-\underline{f}(t, \underline{x}, \bar{x}, u)|^{2}+|\bar{f}(t, \underline{x}, \bar{x}, u)-f(t, x, u, \varrho)|^{2} \leq \\
\beta|x-\underline{x}|^{2}+\beta|\bar{x}-x|^{2}+\alpha
\end{gathered}
$$

for some $\alpha \in \mathbb{R}_{+}, \beta \in \mathbb{R}_{+}$, and

$$
\beta I_{n}-Q+R \preceq 0, R=R^{\mathrm{T}} \succ 0 .
$$

Then in (3), (4) the variables $\underline{x}(t)$ and $\bar{x}(t)$ remain bounded for all $t>0$ and

$$
\underline{x}(t) \leq x(t) \leq \bar{x}(t),
$$

provided that $\underline{x}(0) \leq x(0) \leq \bar{x}(0)$.

The result of Theorem 1 is based on rather restrictive assumption that the matrix $D$ is Metzler. All other assumptions are rather common in the estimation theory (boundedness of the state $x$ and the input $u$ in assumption 1, existence of majorant functions for $f$ from assumption 2, existence of the observer gain $L$ with the corresponding Lyapunov matrix $P$ in assumption 3, Lipschitz continuity or boundedness of $f$, $\bar{f}$ stated in the theorem).

The observer accuracy, i.e. the length of the estimated interval $|\bar{x}(t)-\underline{x}(t)|$, is proportional to the incertitude "size" represented in the set $\Theta$ and the difference $\mid \bar{f}(t, \underline{x}, \bar{x}, u)-$ $\underline{f}(t, \underline{x}, \bar{x}, u) \mid$. It also predefined by the stability margin of the matrix $D(t)$, in order to improve the interval estimation accuracy an $H_{\infty}$ observer can be designed as in [27].

\section{A. Static transformation of coordinates}

For a constant matrix $D$ the assumption that it is Metzler is relaxed in Lemma 2, where it is shown that under conditions of assumption 3 (the matrix $D$ is Hurwitz) there exists a static real similarity transformation matrix $S$ with $S^{-1} D S$ being Hurwitz and Metzler. In our case $D(t, y, u)$ is a matrix variable, an extension of Lemma 2 for this case is presented below.

Lemma 3. [7] Let $D \in \Xi \subset \mathbb{R}^{n \times n}$ be a matrix variable satisfying the interval constraints $\Xi=\left\{D \in \mathbb{R}^{n \times n}: D_{a}-\right.$ $\left.\Delta \leq D \leq D_{a}+\Delta\right\}$ for some $D_{a}^{T}=D_{a} \in \mathbb{R}^{n \times n}$ and $\Delta \in \mathbb{R}_{+}^{n \times n}$. If for some constant $\mu \in \mathbb{R}$ and a diagonal matrix $\Upsilon \in \mathbb{R}^{n \times n}$ the Metzler matrix $R=\mu E_{n}-\Upsilon$ has the same eigenvalues as the matrix $D_{a}$, then there is an orthogonal matrix $S \in \mathbb{R}^{n \times n}$ such that the matrices $S^{T} D S$ are Metzler for all $D \in \Xi$ provided that $\mu>n\|\Delta\|_{\max }$.

The matrix $\mu E_{n}$ has one eigenvalue $\mu n$ and the rest equal zero, the matrix $R$ for $\Upsilon=\rho I_{n}$ with $\rho>\mu n$ is Hurwitz and Metzler. To apply this lemma assume that all its conditions are satisfied.

Assumption 4. Let $D(t, y, u) \in \Xi$ for all $t \geq 0,\|u\| \leq U$ and $\|y\| \leq Y$, where $\Xi=\left\{D \in \mathbb{R}^{n \times n}: D_{a}-\Delta \leq D \leq\right.$ $\left.D_{a}+\Delta\right\}$ for some $D_{a}^{T}=D_{a} \in \mathbb{R}^{n \times n}$ and $\Delta \in \mathbb{R}_{+}^{n \times n}$. Let for some constant $\mu>n\|\Delta\|_{\max }$ and a diagonal matrix $\Upsilon \in \mathbb{R}^{n \times n}$ the Metzler matrix $R=\mu E_{n}-\Upsilon$ has the same eigenvalues as the matrix $D_{a}$.

For $\|\Delta\|_{\max }$ small enough, Assumption 4 becomes a mild one. Under this assumption there is an orthogonal matrix $S \in \mathbb{R}^{n \times n}$ such that the matrices $S^{\mathrm{T}} D(t, y, u) S$ are Metzler for all $D(t, y, u) \in \Xi$. Introduce new state variable $z=S^{\mathrm{T}} x$ $(\|z\| \leq Z$ for some $Z>0$ under Assumption 1), then the system (3) can be rewritten in the new coordinates:

$$
\dot{z}=S^{\mathrm{T}} A(t, y, u) S z+\phi(t, z, u, \varrho),
$$

where $\phi(t, z, u, \varrho)=S^{\mathrm{T}} f(t, S z, u, \varrho)$. Using (1) we have the following relations

$$
\underline{x}=S^{+} \underline{z}-S^{-} \bar{z} \leq x=S z \leq S^{+} \bar{z}-S^{-} \underline{z}=\bar{x},
$$

where $\underline{z} \leq z \leq \bar{z}$ are the interval estimates for the variable $z$. Similarly under assumption 2 we obtain (here $\underline{x}, \bar{x}$ are calculated in (5)):

$$
\begin{gathered}
\underline{\phi}(t, \underline{z}, \bar{z}, u)=S^{+\mathrm{T}} \underline{f}(t, \underline{x}, \bar{x}, u)-S^{-\mathrm{T}} \bar{f}(t, \underline{x}, \bar{x}, u) \leq \\
\phi(t, z, u, \varrho) \leq S^{+\mathrm{T}} \frac{1}{f}(t, \underline{x}, \bar{x}, u)-S^{-\mathrm{T}} \underline{f}(t, \underline{x}, \bar{x}, u) \\
=\bar{\phi}(t, \underline{z}, \bar{z}, u) .
\end{gathered}
$$

In the new coordinates the interval observer takes form similar to (4):

$$
\begin{aligned}
\dot{\dot{z}}= & S^{\mathrm{T}} A(t, y, u) S \underline{z}+\underline{\phi}(t, \underline{z}, \bar{z}, u)+ \\
& S^{\mathrm{T}} L(t, y, u)[y-C(t, u) S \underline{z}], \\
\dot{\bar{z}}= & S^{\mathrm{T}} A(t, y, u) S \bar{z}+\bar{\phi}(t, \underline{z}, \bar{z}, u)+ \\
& S^{\mathrm{T}} L(t, y, u)[y-C(t, u) S \bar{z}] .
\end{aligned}
$$


The applicability conditions for this interval observer are formulated in the following theorem.

Theorem 2. [7] Let assumptions 1, 2, 3 and 4 hold. Let one of the following conditions be satisfied:

1) $|\underline{f}(t, \underline{x}, \bar{x}, u)|<+\infty$ and $|\bar{f}(t, \underline{x}, \bar{x}, u)|<+\infty$ for any $t \geq 0,\|u\| \leq U$ and all $\underline{x} \in \mathbb{R}^{n}, \bar{x} \in \mathbb{R}^{n}$;

2) for any $t \geq 0,\|z\| \leq Z,\|u\| \leq U, \varrho \in \Theta$ and all $\underline{z} \in \mathbb{R}^{n}, \bar{z} \in \mathbb{R}^{n}$

$$
\begin{aligned}
& |\phi(t, z, u, \varrho)-\phi(t, \underline{z}, \bar{z}, u)|^{2}+\mid \bar{\phi}(t, \underline{z}, \bar{z}, u) \\
& -\left.\phi(t, z, u, \varrho)\right|^{2} \leq \beta|z-\underline{z}|^{2}+\beta|\bar{z}-z|^{2}+\alpha
\end{aligned}
$$

for some $\alpha \in \mathbb{R}_{+}, \beta \in \mathbb{R}_{+}$, and

$$
\beta I_{n}-S^{\mathrm{T}} Q S+R \preceq 0, R=R^{\mathrm{T}} \succ 0 .
$$

Then in (3), (5), (6) the variables $\underline{x}(t)$ and $\bar{x}(t)$ are bounded for all $t>0$ and

$$
\underline{x}(t) \leq x(t) \leq \bar{x}(t)
$$

provided that $\underline{z}(0)=S^{+T} \underline{x}(0)-S^{-T} \bar{x}(0), \bar{z}(0)=S^{+T} \bar{x}(0)-$ $S^{-T} \underline{x}(0)$.

This theorem proposes the interval observer for an LTV (LPV) system explicitly skipping the requirement on cooperativity of the closed loop matrix $D$ (according to assumption 3 it is only stable).

\section{B. Time-varying transformation of coordinates}

Another way to relax the Metzler property assumption in Theorem 1 deals with the periodic matrices $D$ applying the Floquet theory. Let $D(t)=D(t, y, u)=A(t, y, u)-$ $L(t, y, u) C(t, u)$ be a periodical matrix with a period $\tau>$ 0 . Denote as $\Phi(t)$ the fundamental matrix of the system $\left(\dot{\Phi}(t)=D(t) \Phi(t)\right.$ for $t \geq 0$ with $\left.\Phi(0)=I_{n}\right)$, then $\Phi(t+T)=\Phi(t) H$, where $H$ is the monodromy matrix and $\Phi(t)=W(t) e^{t B}$ for $B=0.5 \tau^{-1} \ln H^{2}$ and $2 T$-periodical matrix function $W(t)=\Phi(t) e^{-t B}$ for $0 \leq t \leq 2 \tau$.

Assumption 5. There is a Metzler matrix $G$ having the same eigenvalues as the matrix $B$, and the pairs $\left(B, \chi_{1}\right)$ and $\left(G, \chi_{2}\right)$ are observable for some $\chi_{1} \in \mathbb{R}^{1 \times n}, \chi_{2} \in \mathbb{R}^{1 \times n}$.

Under these conditions $G=S B S^{-1}$ for $S=O_{G} O_{B}^{-1}$ where $O_{G}$ and $O_{B}$ are the observability matrices for the pairs $\left(B, \chi_{1}\right)$ and $\left(G, \chi_{2}\right)$ respectively. Define the new coordinates $z=S W^{-1}(t) x$ (again under Assumption 1 there is a constant $Z>0$ such that $\|z\| \leq Z$ since $W(t)$ is a continuous bounded periodic matrix function), then the system (3) equations take the form:

$$
\dot{z}=G z+v(t, z, u, \varrho)+M(t) L(t, y, u) y,
$$

where $v(t, z, u, \varrho)=M(t) f\left(t, M^{-1}(t) z, u, \varrho\right)$ and $M(t)=$ $S W^{-1}(t)$. Under Assumption 2, using the relations (1) we obtain for any $t \geq 0,\|u\| \leq U, \varrho \in \Theta$

$$
\underline{v}(t, \underline{z}, \bar{z}, u) \leq v(t, z, u, \varrho) \leq \bar{v}(t, \underline{z}, \bar{z}, u)
$$

under the condition that $\underline{z} \leq z \leq \bar{z}$ for some $\underline{z}, \bar{z} \in \mathbb{R}^{n}$, where $\underline{v}(t, \underline{z}, \bar{z}, u)=M^{+}(t) \underline{f}(t, \underline{x}, \bar{x}, u)-$ $M^{-}(t) \bar{f}(t, \underline{x}, \bar{x}, u), \bar{v}(t, \underline{z}, \bar{z}, u)=M^{+}(t) \overline{\bar{f}}(t, \underline{x}, \bar{x}, u)-$ $M^{-}(t) \underline{f}(t, \underline{x}, \bar{x}, u)$ with $\underline{x}, \bar{x}$ defined as

$$
\begin{aligned}
& \underline{x}=\left\{M^{-1}(t)\right\}^{+} \underline{z}-\left\{M^{-1}(t)\right\}^{-} \bar{z}, \\
& \bar{x}=\left\{M^{-1}(t)\right\}^{+} \bar{z}-\left\{M^{-1}(t)\right\}^{-} \underline{z} .
\end{aligned}
$$

Then the interval observer equations can be designed as follows:

$$
\begin{aligned}
& \underline{\dot{z}}=G \underline{z}+\underline{v}(t, \underline{z}, \bar{z}, u)+M(t) L(t, y, u) y, \\
& \dot{\bar{z}}=G \bar{z}+\bar{v}(t, \underline{z}, \bar{z}, u)+M(t) L(t, y, u) y .
\end{aligned}
$$

Then the previous theorems admit the following extension.

Theorem 3. Let assumptions 1, 2, 5 hold and the matrix G be Hurwitz (by a proper choice of L, there exist $P=P^{T} \succ 0$ and $Q=Q^{T} \succ 0$ such that $\left.G^{T} P+P G+P^{2}+Q=0\right)$. Let one of the following conditions be satisfied:

1) $|\underline{v}(t, \underline{z}, \bar{z}, u)|<+\infty$ and $|\bar{v}(t, \underline{z}, \bar{z}, u)|<+\infty$ for any $t \geq 0,\|u\| \leq U$ and all $\underline{z} \in \mathbb{R}^{n}, \bar{z} \in \mathbb{R}^{n}$;

2) for any $t \geq 0,\|z\| \leq Z,\|u\| \leq U, \varrho \in \Theta$ and all $\underline{z} \in \mathbb{R}^{n}, \bar{z} \in \mathbb{R}^{n}$

$$
\begin{gathered}
|v(t, z, u, \varrho)-\underline{v}(t, \underline{z}, \bar{z}, u)|^{2}+\mid \bar{v}(t, \underline{z}, \bar{z}, u) \\
-\left.v(t, z, u, \varrho)\right|^{2} \leq \beta|z-\underline{z}|^{2}+\beta|\bar{z}-z|^{2}+\alpha
\end{gathered}
$$

for some $\alpha \in \mathbb{R}_{+}, \beta \in \mathbb{R}_{+}$, and

$$
\beta I_{n}-Q+R \preceq 0, R=R^{\mathrm{T}} \succ 0 .
$$

Then in (3), (5), (6) the variables $\underline{x}(t)$ and $\bar{x}(t)$ are bounded for all $t>0$ and

$$
\underline{x}(t) \leq x(t) \leq \bar{x}(t),
$$

provided that $\underline{z}(0)=M^{+}(t) \underline{x}(0)-M^{-}(t) \bar{x}(0), \bar{z}(0)=$ $M^{+}(t) \bar{x}(0)-M^{-}(t) \underline{x}(0)$.

Proof: Consider the dynamics of the interval estimation errors $\bar{e}=\bar{z}-z, \underline{e}=z-\underline{z}$ :

$$
\begin{aligned}
& \dot{\bar{e}}=G \bar{e}+\bar{v}(t, \underline{z}, \bar{z}, u)-v(t, z, u, \varrho), \\
& \underline{\dot{e}}=G \underline{e}+v(t, z, u, \varrho)-\underline{v}(t, \underline{z}, \bar{z}, u) .
\end{aligned}
$$

Due to assumption 2 for a Metzler matrix $G$, for all $t \geq 0$ the properties $v[t, z(t), u(t), \varrho] \geq \underline{v}[t, \underline{z}(t), \bar{z}(t), u(t)]$, $\bar{v}[t, \underline{z}(t), \bar{z}(t), u(t)] \geq v[t, z(t), u(t), \varrho]$ and

$$
\underline{z}(t) \leq z(t) \leq \bar{z}(t)
$$

are satisfied, provided that $\underline{z}(0) \leq z(0) \leq \bar{z}(0)$ (this condition is true due to the choice of $\underline{z}(0)$ and $\bar{z}(0))$. To prove that the variables $\underline{z}(t), \bar{z}(t)$ are bounded, consider the Lyapunov function $V=\underline{e}^{\mathrm{T}} P \underline{e}+\bar{e}^{\mathrm{T}} P \bar{e}$ and evaluate its derivative:

$$
\begin{aligned}
\dot{V}= & \underline{e}^{\mathrm{T}}\left[G^{\mathrm{T}} P+P G\right] \underline{e}+ \\
& \bar{e}^{\mathrm{T}}\left[G^{\mathrm{T}} P+P G\right] \bar{e}+ \\
& 2 \underline{e}^{\mathrm{T}} P[v(t, z, u, \varrho)-\underline{v}(t, \underline{z}, \bar{z}, u)]+ \\
& 2 \bar{e}^{\mathrm{T}} P[\bar{v}(t, \underline{z}, \bar{z}, u)-v(t, z, u, \varrho)] .
\end{aligned}
$$


Due to conditions of the theorem this equality can be rewritten as follows:

$$
\begin{gathered}
\dot{V} \leq-\bar{e}^{\mathrm{T}} Q \bar{e}-\underline{e}^{\mathrm{T}} Q \underline{e}+ \\
|v(t, z, u, \varrho)-\underline{v}(t, \underline{z}, \bar{z}, u)|^{2}+|\bar{v}(t, \underline{z}, \bar{z}, u)-v(t, z, u, \varrho)|^{2} .
\end{gathered}
$$

If the first condition of the theorem is true, then the terms $|v(t, z, u, \varrho)-\underline{v}(t, \underline{z}, \bar{z}, u)|$ and $|\bar{v}(t, \underline{z}, \bar{z}, u)-v(t, z, u, \varrho)|$ are bounded for any $t \geq 0,\|z\| \leq Z,\|u\| \leq U, \varrho \in \Theta$ and all $\underline{z} \in \mathbb{R}^{n}, \bar{z} \in \mathbb{R}^{n}$. Thus the errors $\bar{e}, \underline{e}$ are bounded by the standard Lyapunov arguments, and so are the variables $\underline{x}$, $\bar{x}$ (from assumption 1 the state $x$ is bounded). If the second condition of the theorem holds, then this inequality becomes:

$$
\dot{V} \leq-\bar{e}^{\mathrm{T}} R \bar{e}-\underline{e}^{\mathrm{T}} R \underline{e}+\alpha,
$$

that implies boundedness of $\underline{z}, \bar{z}$ by the same arguments.

For the periodical case the Floquet theory allows us to transform the problem of interval estimation for a timevarying system into the time-invariant framework.

\section{EXAMPLE}

Consider a time-varying periodical system for $n=2$ :

$$
\begin{aligned}
& \dot{x}=A(t) x+f(t, y, \varrho), y=x_{2}, \\
& A(t)=\frac{\left[\begin{array}{cc}
2 \cos (t)-10 \sin (t)-10.5 & 8 \sin (t)-\cos (t)+10.5 \\
14 & -10.5-2 \sin (t)
\end{array}\right]}{3.5+2 \sin (t)}, \\
& f(t)=\left[\begin{array}{c}
0.1 \sin (2 t) \\
\cos (3 y t)
\end{array}\right]+\varrho,-0.5\left[\begin{array}{l}
1 \\
1
\end{array}\right] \leq \varrho \leq 0.5\left[\begin{array}{l}
1 \\
1
\end{array}\right] .
\end{aligned}
$$

The system is periodical with $\tau=2 \pi$, for simulations we will use

$$
\varrho=-0.5\left[\begin{array}{c}
\tanh \left(x_{2}\right) \cos \left(x_{1}\right) \\
\tanh \left(x_{1}\right) \sin (0.5 t)
\end{array}\right] .
$$

For this choice of $\varrho$ assumptions 1 and 2 are satisfied. For

$$
L=\left[\begin{array}{l}
5 \\
6
\end{array}\right], W(t)=\left[\begin{array}{cc}
2+\sin (t) & 0.5 \\
0.5 & 1
\end{array}\right], S=I_{2}
$$

all other conditions of Theorem 3 (including Assumption 5) are satisfied with

$$
G=\left[\begin{array}{cc}
-5 & 0 \\
6 & -7
\end{array}\right]
$$

The results of interval estimation for the coordinates $x_{1}$ and $x_{2}$ are shown in Fig 1 under assumption that initial conditions for each coordinate belong to the interval $[-0.5,0.5]$.
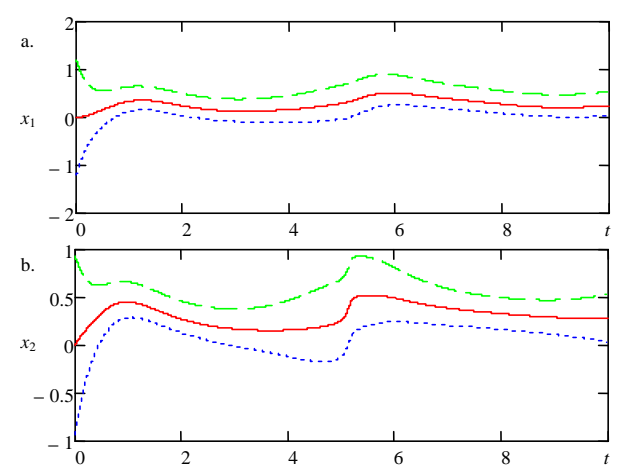

Figure 1. The results of simulations

\section{CONCLUSiON}

The paper is devoted to interval observer design for the LTV systems and the time-varying nonlinear systems in the output canonical form. A static transformation of coordinates is proposed mapping a stable linear uncertain system to another system that is stable and cooperative. In addition, the assumption that there exists an observer gain that makes the estimation error dynamics stable and cooperative is relaxed. The observer gain has to ensure stability of the estimation error as usual, next a transformation of coordinates is proposed, that provides the required cooperativity. The efficiency is shown on examples of computer simulation.

The relaxation of symmetry of the matrix $D_{a}$ introduced in the conditions of the Lemma 3 and the stability conditions used in assumption 4 are the future directions of research.

\section{REFERENCES}

[1] Anstett F., Millerioux G., Bloch G. Polytopic observer design for LPV systems based on minimal convex polytope finding. J. Algorithms and Computational Technology, 3(1), 2009, pp. 23-43.

[2] Bara G.I., Daafouz J., Ragot J., Kratz F. State estimation for affine LPV systems. Proc. 39th IEEE Conf. Decision and Control, CDC, 2000, pp. 4565-4570

[3] Bernard O., Gouzé J.L. Closed loop observers bundle for uncertain biotechnological models. J. Process Control, 14, 2004, pp. 765-774.

[4] Bittanti S., Colaneri P. Periodic Systems: Filtering and Control, ser. Communications and Control Engineering. Springer, 2008.

[5] Blekhman I. Oscillatory strobodynamics - a new area in nonlinear oscillations theory, nonlinear dynamics and cybernetical physics. $C y$ bernetics and Physics, 1(1), 2012, pp. 5-10.

[6] Control and Observer Design for Nonlinear Finite and Infinite Dimensional Systems. Lecture Notes in Control and Information Sciences, Vol. 322, Meurer T., Graichen K., Gilles E.-D. (Eds), Springer, 2005.

[7] Efimov D., Raïssi T., Chebotarev S., Zolghadri A. Interval State Observer for Nonlinear Time Varying Systems, Automatica, 2012, accepted.

[8] Efimov D., Fridman L.M., Raïssi T., Zolghadri A., Seydou R. Interval Estimation for LPV Systems Applying High Order Sliding Mode Techniques. Automatica, 48, 2012, pp. 2365-2371.

[9] Fossen T.I., Nijmeijer H. New Directions in Nonlinear Observer Design. Springer, 1999

[10] Ichalal D., Marx B., Ragot J., Maquin D. An approach for the state estimation of Takagi-Sugeno models and application to sensor fault diagnosis. Proc. 48th IEEE Conf. Decision and Control, CDC/CCC, 2009, pp. 7789-7794.

[11] Jaulin L. Nonlinear bounded-error state estimation of continuous time systems. Automatica, 38(2), 2002, pp. 1079-1082.

[12] Hartman P. Ordinary differential equations. Birkhäuser, 1982. 
[13] Kieffer M., Walter E. Guaranteed nonlinear state estimator for cooperative systems. Numerical Algorithms, 37, 2004, pp. 187-198.

[14] Kieffer M., Walter E. Guaranteed nonlinear state estimation for continuous-time dynamical models from discrete-time measurements. Proc. 5th IFAC Symposium on Robust Control Design, Toulouse, France, 2006.

[15] Köse I.E., Jabbari F. Control of LPV Systems with Partly Measured Parameters. IEEE Trans. Automatic Control, 44(3), 1999, pp. 658-663.

[16] Mazenc F., Bernard O. Interval observers for linear time-invariant systems with disturbances. Automatica, 47(1), 2011, pp. 140-147.

[17] Marcos A., Balas J. Development of linear-parameter-varying models for aircraft. J. Guidance, Control, Dynamics, 27(2), 2004.

[18] Moisan M., Bernard O., Gouzé J.L. Near optimal interval observers bundle for uncertain bio-reactors. Automatica, 45(1), 2009, pp. 291295.

[19] Moore R.E., Kearfott R.B., Cloud M.J. Introduction to Interval Analysis. Philadelphia, SIAM, 2009.

[20] Nonlinear Observers and Applications. Lecture Notes in Control and Information Sciences, Vol. 363, Besançon G. (Ed.), Springer, 2007.

[21] Raïssi T., Efimov D., Zolghadri A. Interval state estimation for a class of nonlinear systems. IEEE Trans. Automatic Control, 57(1), 2012, pp. 260-265.

[22] Raïssi T., Videau G., Zolghadri A. Interval observers design for consistency checks of nonlinear continuous-time systems. Automatica, 46(3), 2010, pp. 518-527.

[23] Shamma J., Cloutier J. Gain-scheduled missile autopilot design using linear parameter-varying transformations. J. Guidance, Control, Dynamics, 16(2), 1993, pp. 256-261.

[24] Smith H.L. Monotone Dynamical Systems: An Introduction to the Theory of Competitive and Cooperative Systems, vol. 41 of Surveys and Monographs, AMS, Providence, 1995.

[25] Tan W. Applications of Linear Parameter-Varying Control Theory. PhD thesis, Dept. of Mechanical Engineering, University of California at Berkeley, 1997.

[26] Tornambe A., Valigi P. Asymptotic stabilization of a class of continuous-time linear periodic systems. Systems and Control Letters, 28(4), 1996, pp. 189-196.

[27] Xie L., de Souza C.E. $H_{\infty}$ State Estimation for Linear Periodic Systems. IEEE Trans. Automatic Control, 38(11), 1993, 1704-1707. 